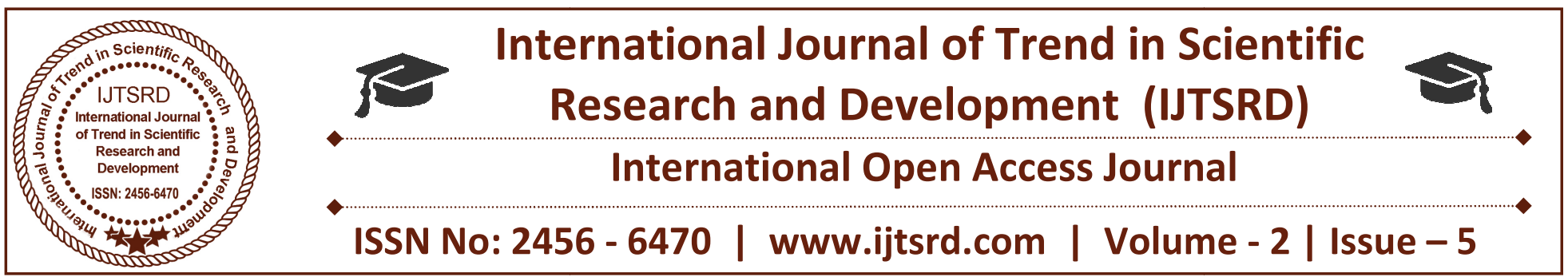

\title{
A Study on Mesh Free Methods: A Different Form of FEM
}

\author{
Vimalkumar P. Palsanawala \\ Lecturer-Mechanical Engineering, \\ Dr. S. \& S. S. Ghandhy College of Engineering \& Technology, Surat, Gujarat, India
}

\section{ABSTRACT}

Roots of Mesh-free methods go back to the seventies. The major difference to finite element methods is that the domain of interest is discretized only with nodes, often called particles. These particles interact via mesh-free shape functions in a continuum framework similar as finite elements do although particle "connectivity" can change over the course of a simulation. This flexibility of mesh-free methods has been exploited in applications with large deformations in fluid and solid mechanics, e.g. to name a few, freesurface flow, metal forming, fracture and fragmentation, to name a few. Though there are a few publications on mesh-free methods formulated in an Eulerian (or ALE) description, e.g. Fries 2005, most mesh-free methods are pure Lagrangian in character. The non negligible advantages of mesh-free methods as compared to finite elements are: their higher order continuous shape functions that can be exploited e.g. for thin shells; higher smoothness; certain advantages in crack propagation problems. The most unhidden drawback of mesh-free methods is probably their higher computational cost, regardless of some instabilities that certain mesh-free methods have. The paper deals with the basic methodology of mesh-free methods along with the mathematics involved.

KEYWORD: discretization, FDM, FEM, FVM, Eigen-value, triangulation, shape function, collocation, SPH

\section{INTRODUCTION}

In the traditional FEM [1, 3], the FDM [5], and the FVM [6], the spatial domain in problem is discretized into meshes. A mesh is defined as any of the open spaces or interstices between the strands of a net that is formed by connecting nodes in a predefined manner.
In FDM, the meshes used are also often called grids; in the FVM, the meshes are called volumes or cells; and in FEM, the meshes are called elements. The terminologies of grids, volumes, cells, and elements carry sometimes certain physical meanings as they are defined for different physical problems. All these grids, volumes, cells, and elements can be termed meshes in accordance with the definition of mesh. The mesh must be predefined to provide a certain relationship between the nodes, which becomes the building blocks of the formulation procedure of the well known conventional numerical methods.

The mesh-free methods establish a system of algebraic equations for the whole problem domain without the use of a predefined mesh, or use easily generable meshes in a much more flexible or "freer', manner. Mesh-free methods essentially use a set of nodes scattered within the problem domain as well as on the boundaries to represent the problem domain and its boundaries. The field functions are then approximated locally using these nodes.

Some of the mesh-free methods are often termed mesh-less method. The ideal requirement for a "mesh-less" method is:

$>$ No mesh is necessary at all throughout the process of solving the problem of given arbitrary geometry governed by partial differential system equations subject to all kinds of boundary conditions.

$>$ The work published so far conclude that the meshfree methods developed so far are not entirely "mesh-less" and fall in one of the following categories:

Methods that require background cells for the integration of system matrices derived from the 
weak form over the problem domain. EFG methods may belong to this category. These methods are practical in many ways, as the creation of a background mesh is generally more feasible and can be much more easily automated using a triangular mesh for $2 \mathrm{D}$ domains and a tetrahedral mesh for 3D domains.

$>$ Methods that require background cells locally for the integration of system matrices over the problem domain. These methods require only a local mesh and are easier to generate.

$>$ Methods that do not require a mesh at all, but that are less stable and less accurate. Local point collocation methods using irregular grids may belong to this category. Automation of nodal selection and improving the stability of the solution are still some of the challenges in these kinds of methods.

$>$ Particle methods that require a predefinition of particles for their volumes or masses. The algorithm will then carry out the analyses even if the problem domain undergoes extremely large deformation and separation. SPH methods belong to this category. This type of method suffers from problems in the imposition of boundary conditions. SPH simulates well the overall behaviors of certain class of problems such as highly nonlinear and momentum-driven problems.

This loose definition of mesh-free method recognizes the fact:

1. Many mesh-free methods (often more robust, reliable, and effective ones) do use some kind of mesh, but the mesh is used in much more flexible and "freer" ways;

2. Most important motivation of developing meshfree methods was to reduce the reliance on the use of "quality" meshes that are difficult or expensive to create for practical problems of complicated geometries

\section{THE IDEA OF MESH FREE METHODS:}

A close examination of the difficulties [1,3] associated with FEM pinpoints at the root of the problem: the heavy and rigid reliance on the use of quality elements that are the building blocks of FEM. A mesh with a predefined connectivity is required to form the elements that are used for both field variable interpolation and energy integration. As long as elements are used in such a rigid manner, the problems shall not be easy to solve. And, the idea of liminating or reducing the reliance on the elements and more flexible ways to make use of mesh has evolved. The concept of element-free, mesh-less, or mesh-free method was proposed, in which the domain of the problem is represented, ideally, only by a set of arbitrarily distributed nodes.

The mesh-free methods have shown great potential for solving the difficult problems mentioned above. Triangular types of mesh that can be much more easily created automatically for complicated $2 \mathrm{D}$ and 3D domains, as shown in Figures 1 and 2. These types of triangular background cells are sufficient for necessary numerical operations in mesh-free methods. This provides flexibility in adding or deleting points/nodes whenever and wherever needed. For stress analysis of a solid domain, for example, there are often areas of stress concentration. One can relatively freely add nodes in the stress concentration area without worrying too much about their relationship with the other existing nodes.

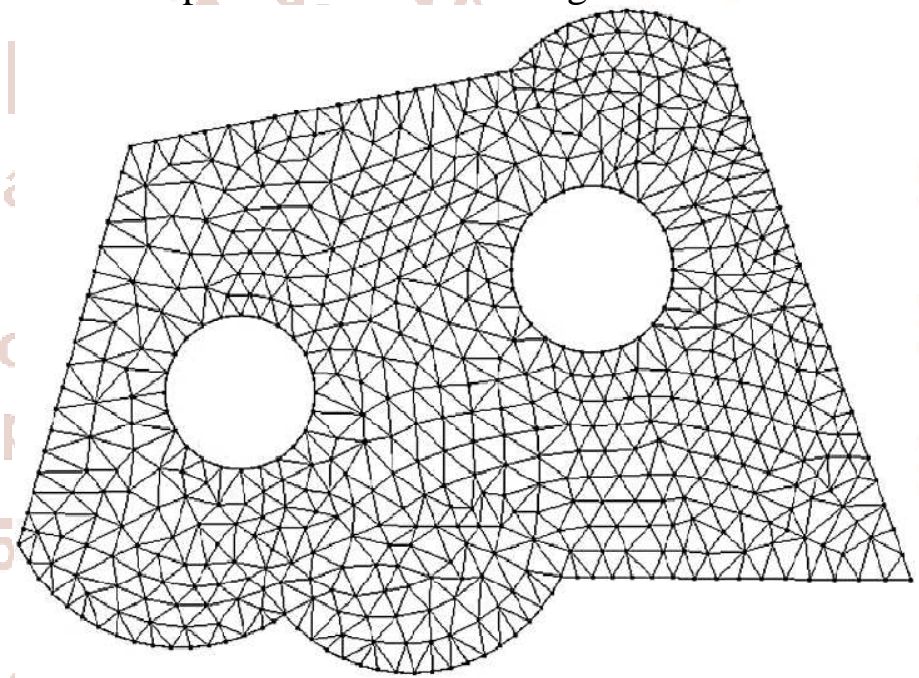

Figure - 1 a triangular mesh of elements or background cells for a complicated 2D domain

In crack growth problems, nodes can be easily added around the crack tip to capture the stress concentration with desired accuracy. This nodal refinement can be moved with a propagation crack through background cells associated with the global geometry. Adaptive meshing for a large variety of problems, 2D or 3D, including linear and nonlinear, static and dynamic stress analysis, can be very effectively treated in mesh-free methods in a relatively simple manner. Because there is no need to create a quality mesh, and the nodes can be created by a computer in a much more automated manner, much of the time an engineer spending on conventional mesh generation can be saved. This can result in to substantial cost and time savings in modeling and simulation projects. 


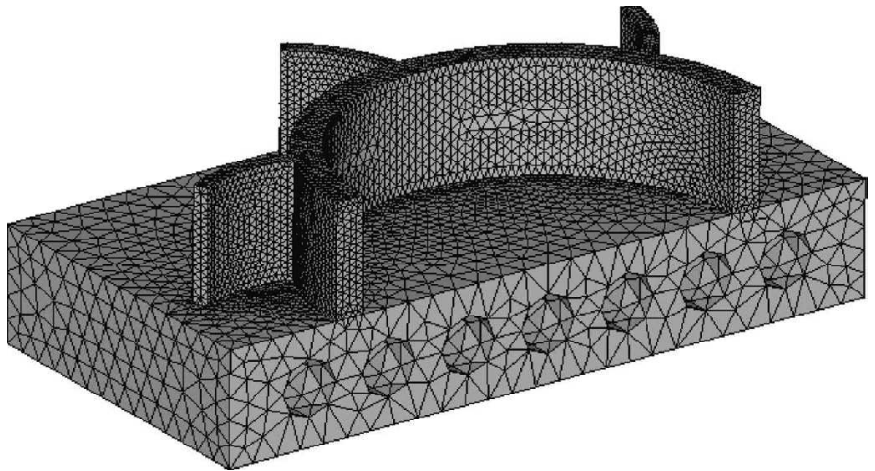

Figure - 2: a tetrahedral mesh of elements or background cells for a complicated 3D domain

\section{BASIC TECHNIQUES FOR MESH-FREE METHODS:}

The general procedure and basic steps for mesh-free methods are presented here under through an example of structural problem.

\section{A. Basic steps:}

Step 1: Domain representation or discretization:

The geometry of the solid or structure is first created in a CAE code or pre processor, and is triangulated to produce a set of triangular type cells with a set of nodes scattered in the problem domain and its boundary. Boundary conditions and loading conditions are then specified for the model. The density of the nodes depends on the presentation accuracy of the geometry, the accuracy requirement of the solution, and the limits of the computer resources available. The nodal distribution is usually not uniform and a denser distribution of nodes is often used in the area where the displacement gradient is larger. Because adaptive algorithms can be used in mesh-free methods, the density is eventually controlled automatically and adaptively in the code of the mesh-free method, we do not have to worry too much about the distribution quality of the initial nodes used in usual situations. In addition, as a mesh-free method, it should not demand too much for the pattern of nodal distribution. It should be workable within reason for arbitrarily distributed nodes. Because the nodes shall carry the values of the field variables in a mesh-free formulation, they are often called field nodes.

Step 2: Displacement interpolation:

The field variable (say, a component of the displacement vector) $\mathrm{u}$ at any point at $\mathrm{x}=(\mathrm{x}, \mathrm{y}, \mathrm{z})$ within the problem domain is approximated or interpolated using the displacements at its nodes within the support domain of the point at $\mathrm{x}$ that is usually a quadrature point, i.e.,

$$
u^{h}(\mathbf{x})=\sum_{i \in S_{n}} \phi_{i}(\mathbf{x}) u_{i}=\Phi(\mathbf{x}) \mathrm{d}_{\mathrm{s}}
$$

Where,

$\mathrm{S}_{\mathrm{n}}$ is the set of local nodes included in a "small local domain" of the point $\mathrm{x}$, such a local domain is called support domain, and the set of local nodes are called support nodes

$u_{\mathrm{i}}$ is the nodal field variable at the $\mathrm{i}^{\text {th }}$ node in the support domain

$\mathrm{d}_{\mathrm{s}}$ is the vector that collects all the nodal field variables at these support nodes

$\phi_{\mathrm{i}}(\mathrm{x})$ is the shape function of the $\mathrm{i}^{\text {th }}$ node created using all the support nodes in the support domain and is often called nodal shape function

A support domain of a point $\mathrm{x}$ determines the number of nodes to be used to approximate the function value at $x$. A support domain can be weighted using functions that vanish on the boundary of the support domain, as shown in Figure-3. It can have different shapes and its dimension and shape can be different for different points of interest $\mathrm{x}$, as shown in Figure-4. The shapes most often used are circular or rectangular, or any shape to include desired supporting nodes. The concept of support domain works well if the nodal density does not vary too drastically in the problem domain. However, in solving practical problems, such as problems with stress singularity, the nodal density can vary drastically. The use of a support domain based on the current point of interest can lead to spatially biased selection of nodes for the construction of shape functions.

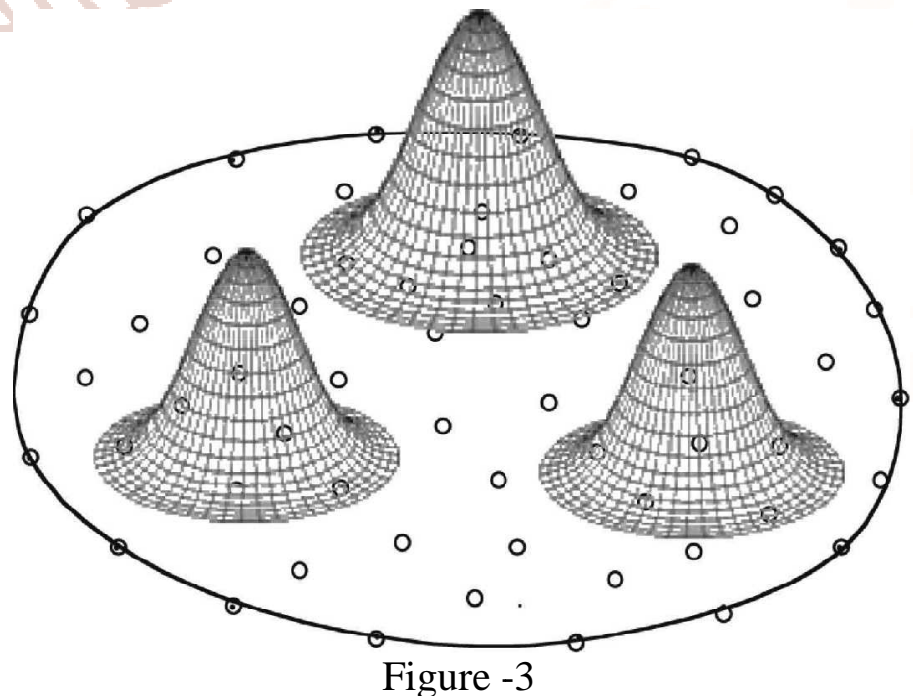




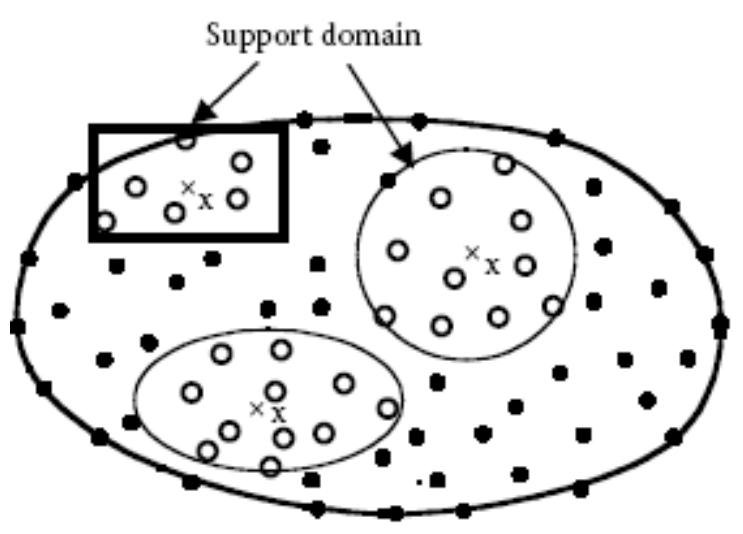

Figure - 4

obtained. The strain and stress can then be retrieved using strain- displacement relations and constitutive equations. A standard linear algebraic equation solver, such as a Gauss elimination method, LU decomposition method, and iterative methods, can be used.

2. For free-vibration and buckling problems, Eigen values and corresponding eigenvectors can be obtained using the standard Eigen-value equation solvers. Some of the commonly used methods are the following:

Jacobi's method

In extreme situations, all the nodes used could be located on one side only, and the shape functions so constructed can result in serious error, due to extrapolation. To prevent this kind of problem, the concept of influence domain of a node should be used. The concept of influence domain is explained later on in the discussion. The interpolation, defined in Equation (1), is generally performed for all the components of all the field variables in the same support domain. For an example taking a 3D solid mechanics problem, the displacement is usually chosen as the field variable, and the displacement would have three components: displacements in the $\mathrm{x}$ , $y$-, and z-directions. The same shape function is used for all three displacement components in the support domain of the same point. However, there are situations where different shape functions are used for different field variables. For example, for bending problems of beams, plates, and shells, it is advantageous to use different shape functions, respectively, for deflection and rotation, in overcoming the so-called shear and membrane locking issues.

Step 3: Formation of system equations:

The discrete equations of a mesh-free method can be formulated using the shape functions and weak or weakened-weak forms. These equations are often written in nodal matrix form and are assembled into the global system matrices for the entire problem domain. The procedures for forming system equations are different for different mesh-free methods.

Step 4: Solving the global mesh-free equations:

Solving the set of global mesh-free equations, we obtain solutions for different types of problems.

1. For static problems, the displacements at all the nodes in the entire problem domain are first

$>$ Given's method

The bisection method

Inverse iteration

Subspace iteration

Lanczos method

3. For dynamics problems, the time history of displacement, velocity, and acceleration are to be obtained. The following standard methods of solving dynamics equation systems can be used:

The modal superposition method can be used for vibration types of problems and problems of far field response to low speed impact with many load cases.

For problems with a single load or few loads, the direct integration method can be used, which uses the FDM for time stepping with implicit and explicit approaches.

The implicit method is more efficient for relatively slow phenomena of vibration types of problems.

The explicit method is more efficient for very fast phenomena, such as impact and explosion.

For computational fluid dynamics problems, the discretized system equations are basically nonlinear, and one needs an additional iteration loop to obtain the results.

\section{B. Triangulation:}

Consider a d-dimensional problem domain of $\Omega \in \mathrm{R}^{\mathrm{d}}$ bounded by $\Gamma$. By default, we speak of "open", domain that does not include the boundary of the domain. When we refer to a "closed" domain we will specifically use a box: $\Omega=\Omega \cap \Gamma$.

Triangulation is the most flexible way to create background triangular cells for mesh-free operations. The process can be almost fully automated for $2 \mathrm{D}$ and 
even 3D domains with complicated geometry. Therefore, it is used in most commercial pre processors using processes such as the widely used Delaunay triangulation.

Concept of the Influence Domain:

The support domain is defined as a domain in the vicinity of a point of interest $\mathrm{x}_{\mathrm{Q}}$ that can be, but does not have to be, at a node. It is used to include the nodes for shape function construction for $\mathrm{x}_{\mathrm{Q}}$. The extended concept of the support domain means a particular way to select those nodes, not necessarily just by distance.

The influence domain is defined as a domain that a node exerts an influence upon. It works well for very irregularly distributed nodes. Influence domains are defined for each node in the problem domain, and they can be different from node to node to represent the area of influence of the node, as shown in Figure6.

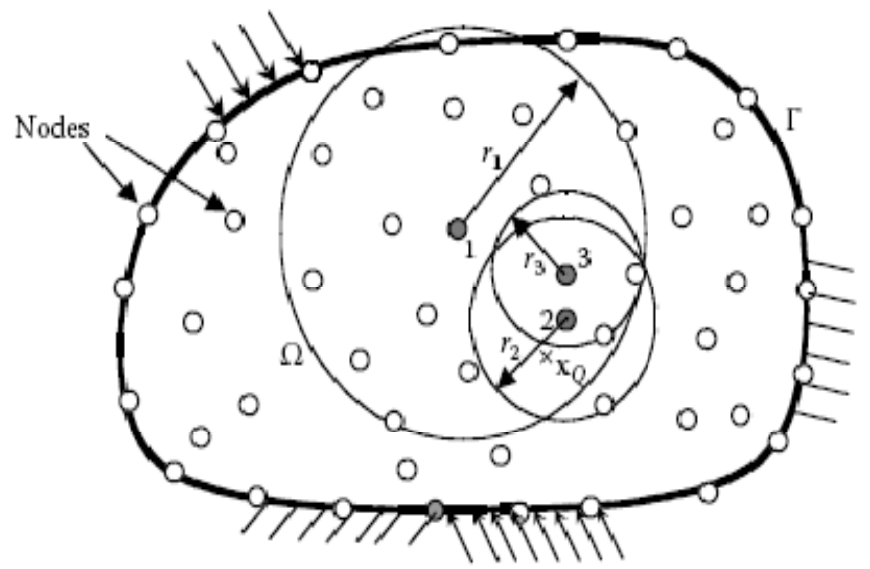

Figure - 6: Influence domains of nodes

In constructing shape functions for point at $\mathrm{xQ}$ (marked with $\mathrm{x}$ ), nodes whose influence domains covers $\mathrm{x}$ are to be used for construction of shape functions. For example, nodes 1 and 2 are included, but node 3 is not included.

Node 1 has an influence radius of $r_{1}$, and node 2 has an influence radius of $r_{2}$, etc. The node will be involved in the shape function construction for any point that is within its influence domain. For example, in constructing the shape functions for the point marked with $\mathrm{x}$ at point $\mathrm{x}_{\mathrm{Q}}$ (see Figure - 6), nodes 1 and 2 will be used, but node 3 will not be used.

The formula to compute the dimension of the influence domain of that node is

$$
r_{I}=\alpha_{s} \sqrt{\frac{2}{n_{I}} \sum_{i=1}^{n_{I}} a_{i}}
$$

Where

$r_{I}$ is the radius of the influence domain of node I

$\mathrm{n}_{\mathrm{I}}$ is the number of surrounding triangular cells

$\mathrm{a}_{\mathrm{i}}$ is the area of the ith cell

$\alpha_{\mathrm{s}}$ is a constant scaling with the domain size

The fact is that the dimension of the influence domain, which can be different from node to node, allows some nodes to have further influence than others and prevents unbalanced nodal distribution for constructing shape functions.

As shown in Figure - 6, node 1 is included for constructing shape functions for the point at point $\mathrm{x}_{\mathrm{Q}}$, but node 3 is not included, even though node 3 is closer to $\mathrm{x}_{\mathrm{Q}}$ compared with node 1 .

\section{T-Schemes for Node Selection:}

Since the background cells are needed for integration for weak or weakened-weak form mesh-free methods, background cells are often already made available. Therefore, it is natural to make use of them also for the selection of supporting nodes for shape function construction. Background cells of triangular type generated by triangulation have been found most practical, robust, reliable, and efficient for local supporting node selection. Triangular cell-based node selection schemes are termed as T-schemes, and are listed in Table 1.

Table 1-T-Schemes for Node Selection Based on Triangular Background Cells

\begin{tabular}{|c|c|c|}
\hline Name & $\begin{array}{l}\text { Node Selection for Interpolation at Any Point } \\
\qquad \text { in a Home Cell }\end{array}$ & $\begin{array}{l}\text { Application/Types } \\
\text { of Shape Functions }\end{array}$ \\
\hline T3-scheme & Three nodes of the home cell & $\begin{array}{l}\text { 2D domain } \\
\text { 3D domain surface } \\
\text { PIM }\end{array}$ \\
\hline T6/3-scheme & $\begin{array}{l}\text { For an interior home cell, three nodes of the home cell } \\
\text { and three remote nodes of the three neighboring cells } \\
\text { For a boundary home cell, three nodes of the home cell }\end{array}$ & $\begin{array}{l}\text { 2D domain } \\
\text { 3D domain surface } \\
\text { PIM, RPIM }\end{array}$ \\
\hline T6-scheme & $\begin{array}{l}\text { For an interior home cell, three nodes of the home cell } \\
\text { and three remote nodes of the three neighboring cells } \\
\text { For a boundary home cell, three nodes of the home cell, } \\
\text { two (or one) remote nodes of the neighboring cells plus } \\
\text { one (or two) field node which is nearest to the centroid } \\
\text { of the home cell }\end{array}$ & $\begin{array}{l}\text { 2D domain } \\
\text { 3D domain surface } \\
\text { PIM, RPIM }\end{array}$ \\
\hline T4-scheme & Four nodes of the home tetrahedral cell & 3D domain, PIM \\
\hline T2L-scheme & $\begin{array}{l}\text { Nodes of the home cell plus one layer of nodes of the cells } \\
\text { connected to the home cell nodes (two layers of nodes } \\
\text { are selected) }\end{array}$ & $\begin{array}{l}\text { 2D domain } \\
\text { 3D domain surface } \\
\text { 3D domain }\end{array}$ \\
\hline
\end{tabular}


In the definition of types of T-schemes, a home cell refers to the cell which hosts the point of interest (usually the quadrature sampling point). An interior home cell is a home cell that has no edge on the boundary of the problem domain and a boundary home cell is a home cell which has at least one edge on the boundary. A neighboring cell of a home cell refers to the cell which shares one edge with the home cell.

Figure-7 shows the background triangular cells for 2D domain. The node selection is then performed as follows.

\section{T3-Scheme:}

In the T3-scheme, we simply select three nodes of the home cell of the point of interest. As illustrated in Fig-7 (a), no matter the point of interest $x$ located in an interior home cell (cell i) or a boundary home cell (cell $j$ ), only the three nodes of the home cell $\left(i_{1} \_i_{3}\right.$ or $\left.\mathrm{j}_{1} \_\mathrm{j}_{3}\right)$ are selected. T3-scheme is used only for creating linear PIM (Point Interpolation Method) shape functions by using polynomial basis functions. Note that the linear PIM shape functions so constructed are exactly the same as those in FEM using linear triangular elements.

T4-Scheme:

T4-scheme is the analogy of the T3-scheme, but of node selection for 3D domains with tetrahedral background cells.

\section{T6/3-Scheme:}

The $T 6 / 3$-scheme selects six nodes to interpolate a point of interest located in an interior cell and three nodes for those located in boundary home cells. As illustrated in Figure-7 (b), when the point of interest $\mathrm{x}_{\mathrm{Q}}$ is located in an interior home cell (cell $\mathrm{i}$ ), we select six nodes: three nodes of the home cell $\left(\mathrm{i}_{1} \mathrm{i}_{3}\right)$ and another three nodes located at the remote vertices of the three neighboring cells $\left(i_{4} i_{6}\right)$. When the point of interest at $\mathrm{x}_{\mathrm{Q}}$ is located in a boundary home cell (cell $\mathrm{j}$ ), we select only three nodes of the home cell, i.e., $\mathrm{j}_{1} \_\mathrm{j}_{3}$.

T6/3-scheme was purposely devised for creating highorder PIM shape functions, where quadratic interpolations are performed for the interior home cells and linear interpolations for boundary home cells. This scheme was first used in the NS-PIM [11]. It can not only successfully overcome the singular problem but also improve the efficiency of the method.
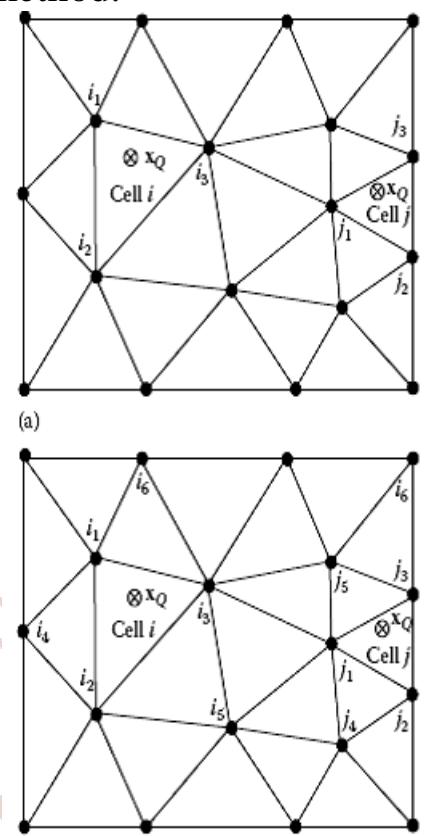

(c)
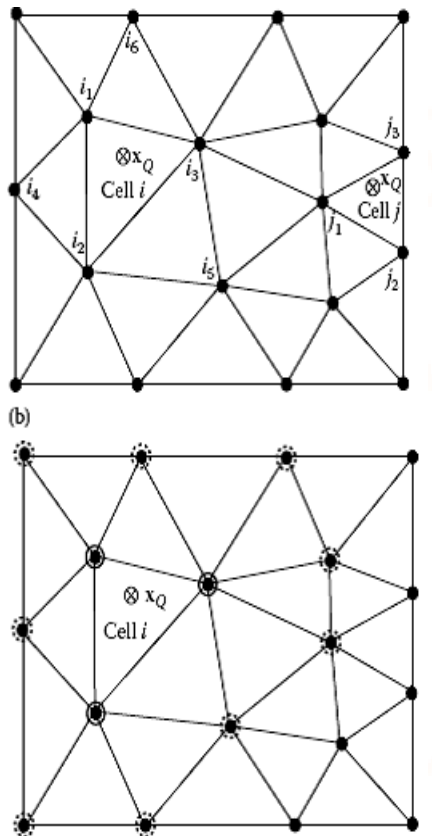

(d)

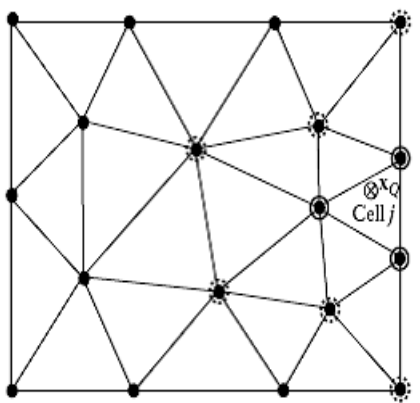

(e)

O First layer of nodes Second layer of nodes

Figure - 7 (a) T3 Scheme, (b) T6/3-Scheme, (c) T6Scheme, (d) \& (e) T2L-Scheme

T6-Scheme:

Similar to T6/3-scheme, T6-scheme, as shown in Figure-7 (c), also selects six nodes for an interior home cell: three nodes of the home cell and three vertexes at the remote vertices of the three neigh boring cells $\left(i_{1} i_{6}\right.$ for cell $\left.i\right)$. However, for a boundary cell (cell j), T6-scheme still selects six nodes: three nodes of the home cell $\left(j_{1} \_j_{3}\right)$, two remote nodes of the neigh boring cells $\left(j_{4}\right.$ and $\left.j_{5}\right)$, and one field node (j6) which is nearest to the centroid of the home cell excepting the five nodes that have been selected.

T6-scheme is purposely devised for constructing radial PIM (RPIM) shape functions on considering both accuracy and efficiency. Different from T6/3scheme, this scheme selects six nodes for all home cells containing the point of interest. 
T2L-Scheme:

T2L-scheme selects two layers of nodes to perform interpolation based on triangular meshes. As shown in Figure-7 (d) and (e), the first layer of nodes refers the three nodes of the home cell, and the second layer contains those nodes which are directly connected to the three nodes of the first layer. This scheme usually selects much more nodes than the T6-scheme and leads to more time consumption. We can use this scheme to create RPIM shape functions with high order of consistence and for extremely irregularly distributed nodes. Such RPIM shape functions can also be used for strong form mesh-free method methods where higher order of consistence is required. T2L-scheme can also be used for creating moving least squares (MLS) shape functions.

\section{REFERENCES}

1. Lucy, L., A numerical approach to testing the fission hypothesis, Astronomical J., 82, 10131024, 1977.

2. Gingold, R. A. and Monaghan, J. J., Smooth particle hydrodynamics: Theory and applications to non-spherical stars, Mon. In Notices $R$. Astronomical Soc., 181, 375-389, 1977.

3. Monaghan, J. J., Why particle methods work, Siam J. Sci. Stat. Comput., 3(4), 423-433, 1982.

4. Liu, M. B., Liu, G. R., and Zong, Z., An overview on smoothed particle hydrodynamics, Int. J. Comput. Methods, 5(1), 135-188, 2008.

5. Zhou, C. E., Liu, G. R., and Lou, K. Y., Threedimensional penetration simulation using smoothed particle hydrodynamics, Int. J. Comput. Methods, 4(4), 671-691, 2007.

6. Liu, G. R. and Liu, M. B., Smoothed Particle Hydrodynamics-A Mesh free Practical Method, World Scientific, Singapore, 2003.

7. Liu, G. R., Zhang, J., and Lam, K. Y., A gradient smoothing method (GSM) with directional correction for solid mechanics problems, Comput. Mech., 41, 457-472, 2008.

8. Liu, G. R. and Xu, G. X., A gradient smoothing method (GSM) for fluid dynamics problems, Int. J. Numerical Methods Fluids, 56(10), 1101-1133, 2008.

9. Xu, G. X., Liu, G. R., and Lee, K. H., Application of gradient smoothing method (GSM) for steady and unsteady incompressible flow problems using irregular triangles, Submitted to Int. J. Numerical Methods Fluids, 2008.

10. Xu, G. X. and Liu G. R., An adaptive gradient smoothing method (GSM) for fluid dynamics problems, Int. J. Numerical Methods Fluids, accepted, 2008.

11. Liu, G. R., Liu, M. B., and Lam, K. Y., A general approach for constructing smoothing functions for meshfree methods, presented at Ninth International Conference on Computing in Civil and Building Engineering, Taipei, China, April 35, 2002, pp. 431-436.

12. Liu, W. K., Adee, J., and Jun, S., Reproducing kernel and wavelet particle methods for elastic and plastic problems, in Advanced Computational Methods for Material Modeling, Benson, D. J., ed., AMD 180=PVP 268 ASME, New Orleans, LA, 1993, pp. 175-190.

13. Liu, W. K., Jun, S., and Zhang, Y., Reproducing kernel particle methods, Int. J. Numerical Methods Fluids, 20, 1081-1106, 1995.

14. Liu, W. K., Chen, Y., Chang, C. T., and Belytschko, T., Advances in multiple scale kernel particle methods, Comput. Mech., 18, 73-111, 1996.

15. Liu, W. K., Jun, S., Sihling, D. T., Chen, Y. J., and Hao, W., Multiresolution reproducing kernel particle method for computational fluid dynamics, Int. J. Numerical Methods Fluids, 24, 1-25, 1997.

16. Liu, W. K., Jun, S., Sihling, D. T., Chen, Y. J., and Hao, W., Multi resolution reproducing kernel particle method for computational fluid dynamics, Int. J. Numerical Methods Fluids, 24, 1391-1415, 1997.

17. Liu, W. K., Li, S. F., and Belytschko, T., Moving least-square reproducing kernel methods: I Methodology and convergence, Comput. Methods Appl. Mech. Eng., 143, 113-154, 1997.

18. Chen, J. S., Pan, C., Wu, C. T., and Liu, W. K., Reproducing kernel particle methods for large deformation analysis of nonlinear structures, Comput. Methods Appl. Mech. Eng., 139, 195228, 1996.

19. Uras, R. A., Chang, C. T., Chen, Y., and Liu, W. K., Multi resolution reproducing kernel particle methods in acoustics, J. Comput. Acoust., 5(1), 71-94, 1997. 
20. Lancaster, P. and Salkauskas, K., Surfaces generated by moving least squares methods, Math. Comput., 37, 141-158, 1981.

21. Cleveland, W. S., Visualizing Data, AT\&T Bell Laboratories, Murray Hill, NJ, 1993.

22. Nayroles, B., Touzot, G., and Villon, P., Generalizing the finite element method: Diffuse approximation and diffuse elements, Comput. Mech., 10, 307-318, 1992.

23. Belytschko, T., Lu, Y. Y., and Gu, L., Elementfree Galerkin methods, Int. J. Numerical Methods Eng., 37, 229-256, 1994.
24. Krongauz, Y. and Belytschko, T., Enforcement of essential boundary conditions in mesh less approximations using finite elements, Comput. Methods Appl. Mech. Eng., 131(1-2), 133-145, 1996.

25. Belytschko, T., Gu, L., and Lu, Y. Y., Fracture and crack growth by element free Galerkin methods, Model. Simulations Mater. Sci. Eng., 2, 519-534, 1994.

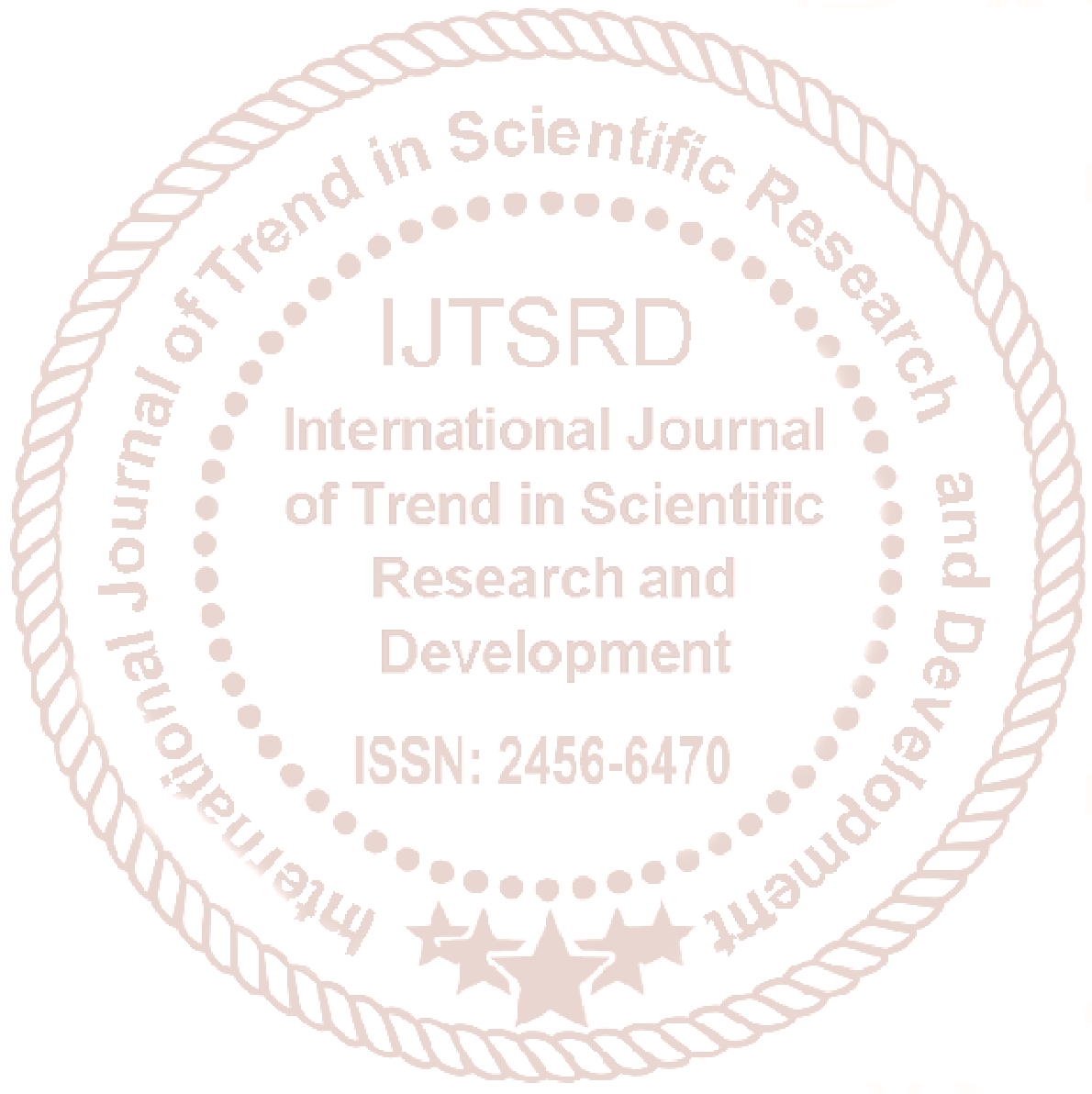

